\title{
Aplicación Web para la Gestión de la información en la Red Integral de Bienestar Social
}

\author{
Web Application for Information Management in the Integral Social Welfare \\ Network
}

MENA-CASTILLO, Lilia Margarita†*, MOLINA-CEPEDA, Isaac Neftali y GARCÍA-ANDAZOLA, Saúl

Instituto Tecnológico Superior de Nuevo Casas Grandes

ID $1^{\text {er }}$ Autor: Lilia Margarita, Mena-Castillo / ORC ID: 0000-0001-9255-5991, CVU CONACYT ID: 472653

ID $1{ }^{\text {er }}$ Coautor: Isaac Neftali, Molina-Cepeda / ORC ID: 0000-0002-6557-6612, CVU CONACYT ID: 808687

ID $2^{\text {do }}$ Coautor: Saúl, García-Andazola / ORC ID: 0000-0002-1803-5936, CVU CONACYT ID: 848918

DOI: $10.35429 / J I T C .2019 .9 .3 .24 .30$

Recibido 06 de Julio, 2019; Aceptado 30 de Septiembre, 2019

\begin{abstract}
Resumen
La información se ha convertido en un recurso fundamental en las empresas y al estar acompañada de las Tecnologías de la Información y la Comunicación, se ha vuelto más significativo el utilizarlas, ya que se pueden lograr grandes cambios en las empresas. Mediante su gestión por medio de herramientas tecnológicas, se pueden tomar decisiones más fácilmente, ya que existen diversas formas de representar la información, para que sea más clara y objetiva, además de acceder a ella desde cualquier lugar de manera confiable. El presente trabajo muestra el desarrollo de un sitio web que se le realizó a la asociación Red Integral de Bienestar Social (RIBS), la cual trabaja con 20 asociaciones más y necesita tener una comunicación y retroalimentación más estrecha con ellas en cuanto a información pública y privada, según sea el caso. Se utilizó la metodología scrum, para el desarrollo del sitio web y el lenguaje de programación fue PHP, utilizando el framework Laravel y para la gestión de la información se utilizó el gestor de MySQL.
\end{abstract}

\section{Asociación, Tecnologías, Sitio Web}

\begin{abstract}
Information has become a fundamental resource in companies and, being accompanied by Information and Communication Technologies, it has become more significant to use them, since great changes can be achieved in companies. Through its management through technological tools, decisions can be made more easily, since there are different ways of representing information, to make it more clear and objective, as well as to access it from any place in a reliable manner. This work shows the development of a website that was made to the association Integral Social Welfare Network (RIBS), which works with 20 other associations and needs to have a closer communication and feedback with them in terms of public information and private, as the case may be. The scrum methodology was used, for the development of the website and the programming language was PHP, using the Laravel framework and for the management of the information the MySQL manager was used.
\end{abstract}

\section{Association, Technologies, Website}

Citación: MENA-CASTILLO, Lilia Margarita, MOLINA-CEPEDA, Isaac Neftali y GARCÍA-ANDAZOLA, Saúl. Aplicación Web para la Gestión de la información en la Red Integral de Bienestar Social. Revista de Tecnologías de la Información y Comunicaciones. 2019. 3-9: 24-30

\footnotetext{
* Correspondencia del Autor (Correo electrónico: Imena@itsncg.edu.mx)

$\dagger$ Investigador contribuyendo como primer autor.
} 


\section{Introducción}

En la era digital en la cual estamos viviendo, cada vez encontramos que las empresas incorporan los recursos de Tecnología de Información (TI) como elemento clave de su estrategia organizacional. No solo las empresas que han nacido en esta era digital, sino que también las empresas provenientes de ambientes tradicionales (pre digital) se encuentran utilizando estos recursos y modificando sus procesos o incluso redefiniendo sus modelos de negocio. Dichas Tecnologías de Información utilizadas para mejorar los procesos en las empresas son esenciales para apoyar la generación de competitividad en las organizaciones.

La información es una parte esencial e imprescindible en todo proceso de una organización, es la médula para todo crecimiento económico y social; actualmente permite niveles superiores de competitividad debido a la demanda permanente $y$ cada vez mayor información. [1]

Las organizaciones están conscientes de que los recursos de TIC son herramientas de cambio en la realización de sus actividades, ya que les permiten minimizar tiempo, mejorar integridad y seguridad de la información, disminuir información redundante y permitir por tanto que la información y el conocimiento que se genera cumplan con sus objetivos dentro del plan estratégico y operativo de las organizaciones.

Es importante que éstas definan clara y detalladamente qué quieren ser para que entonces se analice de qué forma serán incorporados los recursos de Tecnologías de Información y Comunicación. Además, se debe de considerar que muchas empresas están comenzando a evolucionar sus estructuras organizacionales a estructuras virtuales donde las tecnologías de información son el corazón. Sin embargo, es importante aprender de los errores y experiencias previas de manera tal que estas nuevas formas de organización puedan lograr su propósito. [2]

Además, la presencia en línea es vital para una empresa pues es un campo muy competitivo, donde las empresas que ya cuentan con un sitio web están abarcando mercados potenciales.
Seguramente hay muchas empresas que ya tienen en mente hacer su sitio web, pero la mayoría no sabe cómo puede iniciar el proceso sin tener que gastar en exceso. Es por ello que un sitio web debe ser profesionalmente desarrollado para que dé los resultados esperados a la empresa, ya que este será la imagen de la empresa en la red. También las formas de comunicarse con los clientes se revolucionan, con las diferentes opciones, desde correos electrónicos, chats, boletines de información concreta y objetiva en el sitio. Se mejora la información y comunicación con los clientes actuales o futuros, a proveedores, socios $\mathrm{y}$ colaboradores, empleados y sobre todo con el entorno social [3].

\section{Definición del Problema}

En enero de 2018, se realizó un acercamiento por parte de la asociación "Red Integral de Bienestar Social" (RIBS), al Instituto Tecnológico Superior de Nuevo Casas Grandes, ya que presentaba una problemática con la difusión y control de su información, por lo que se estaba generando que algunas de sus actividades fueran deficientes, por ejemplo en el proceso de la creación de nuevos eventos, ya que esta asociación consta de varias agrupaciones y al momento de crear un nuevo evento o junta, la encargada de la asociación RIBS, informaba por correo y/o vía telefónica sobre el evento y temas a tratar, a cada una de las asociaciones.

Otra problemática que se detecto fue la difusión en el control de las actas realizadas cuando se reúnen y sobre todo de las finanzas que llevan en la asociación, por lo que las evidencias para la transparencia se ven un poco informales y esto puede afectar en la imagen de la asociación y esto podrían generar la pérdida de recursos.

En base a lo anterior el Objetivo General del proyecto es desarrollar y fortalecer el uso de medios electrónicos en RIBS, para facilitar y agilizar la información entre las asociaciones por medio de un sitio Web de fácil acceso y amigable. Además, con esta investigación se pretende contribuir un poco con la sociedad, vinculando y transfiriendo los conocimientos de los estudiantes en un problema del entorno real. 


\section{Material y Método}

La investigación se llevó a cabo en la asociación Red integran de Bienestar Social, de Nuevo Casas Grandes, Chihuahua, la cual está conformada por 20 asociaciones. La muestra poblacional fue aproximadamente del $25 \%$ de las asociaciones las cuales fueron: la asociación RIBS, el Instituto Blas Pascal, Centro de Formación y Capacitación en Apoyo a Necesidades Educativas Especiales, Fundación Cambiando Destinos, Centro de Rehabilitación de Nuevo Casas Grandes, Formación y Desarrollo Familiar de NCG. Se decidió tomar solo el $25 \%$ de las asociaciones ya que se tenía que ir constantemente al lugar donde se encontraban ubicadas las instalaciones de dichas asociaciones y utilizar parte del tiempo de trabajo de las personas involucradas en el proyecto.

Las variables a considerar son los procesos para comunicar información a otras asociaciones y el tiempo que se utiliza para comunicar la información. Además de los procesos que realizan las asociaciones para acceder a cierta información y el tiempo que se toman las asociaciones para acceder a la información.

Es de suma importancia definir el enfoque de la investigación ya que "se denomina enfoque a la manera de apreciar la investigación según la naturaleza de las variables. Teniendo en cuenta esta naturaleza, el estudio puede ser cuantitativo si las variables que se proponen estudiar son de naturaleza cuantitativa, es decir, se miden con cantidades. En cambio, un estudio se verá como cualitativo si las variables son cualitativas, lo cual quiere decir que se miden con cualidades. Será mixto el estudio si unas variables son cualitativas y otras cuantitativas" [4]. En este caso el enfoque es cuantitativo, ya que se medirán variables cuantificables.

En el proyecto participaron varios alumnos del Instituto Tecnológico Superior de Nuevo Casas Grandes, de la carrera de Ingeniería en Sistemas Computacionales de octavo semestre, bajo la supervisión de varios docentes de la misma institución, los alumnos que participaron en este proyecto son: Iván Torres Mercado, Carlos Torres García y Ramiro González Montiel, los cuales colaboraron en la parte del análisis y la programación de un sitio web.
Fueron varias las materias del semestre que se vieron involucradas en el desarrollo del proyecto, el cual fue un gran estímulo para los jóvenes al realizar un proyecto real para una empresa externa a la institución en la cual estudian. La metodología de desarrollo del software que se utilizó para la creación del sistema fue de desarrollo ágil, y se seleccionó la metodología Scrum, ya que Antonio Martel menciona en su libro Gestión práctica de proyectos con scrum, que esta metodología "es un framework o conjunto de buenas prácticas para la gestión de proyectos. Se basa en un estudio realizado sobre los procesos de desarrollo utilizados con éxito en Canon, Xerox, Honda o HP (que en los años 80 eran las Google o Apple de ahora). En este estudio se comprobó que estos equipos partían de unos requisitos muy generales para sus productos y que además debían salir al mercado en poco tiempo. Eran equipos altamente productivos que seguían unos patrones muy similares" [5].

\section{Requerimientos}

Se realizaron algunas visitas a la asociación RIBS y a las asociaciones muestra, para recolectar información acerca de las asociaciones y procedimientos que realizaban comúnmente, además, se desarrolló una entrevista con la encargada de la asociación para validar la información recabada y complementar la información. Con la información recabada se obtuvo una idea más clara de las necesidades que deben ser cubiertas por el proyecto. También, se comentó que se realizaba la difusión de actas de cada mes por medio de correo electrónico por este medio envían información de convocatorias, el orden del día, las finanzas, mensajes y nuevos eventos.

\section{Análisis}

En base a lo anterior se realizó un análisis de la problemática que tiene la asociación Ribs y se planteó una propuesta sobre la posible implementación de un sitio web, esto con el fin de que sea más eficaz ver la información de cada junta y que la asociación pueda realizar sus actividades de acuerdo con el orden del día. 
El sitio web contendrá una pantalla de administrador y una pantalla para cada usuario administrador de las demás asociaciones esto con el fin de dar a conocer la información financiera, las convocatorias, mensajes personales y eventos, de esta manera tener un mayor rendimiento y cada asociación obtendrá la información perteneciente a los programas que maneja Ribs. Además, se realizó un análisis de factibilidad técnico, económico y operacional para que la implementación del sitio sea factible para la asociación.

La asociación Ribs estuvo de acuerdo con la propuesta, por lo que se empezó a trabajar con la calendarización de las actividades del proyecto. Guillermo Pantaleo, menciona en su libro Calidad en el desarrollo de software que "es interesante fijar puntos de observación en el cronograma del proyecto orientados a informar el estado y avance y tomar decisiones al respecto. Estos puntos de observación son informados en algún reporte y analizados con todos los involucrados" [6]. Para esta calendarización de actividades se utilizó la herramienta MS-Project, creando una Gráfica de Gantt con las actividades, involucrados en el proyecto y las fechas de entrega de tareas.

\section{Diseño}

A continuación, se realizó la maquetación del sitio web, es decir, se diseñaron las pantallas de entrada y salida de datos, luego se presentó un avance a la asociación, para conocer si era de su agrado las pantallas en cuanto a amigabilidad y diseño, lo cual fue muy satisfactorio.

También se realizó el diseño de la base de datos, así como las tablas, campos y atributos. Es el punto más importante en el diseño, ya que la base de datos cuenta con muchas relaciones, llaves primarias y llaves foráneas. Se creó la conexión en el servidor local utilizando el servidor de MySQL para lograr conectar la base de datos con el sitio web y que todo funcione correctamente. José Manuel Espinoza autor del libro Informática Industrial, menciona que "El diseño de una página web requiere, en muchas ocasiones, el uso de imágenes dinámicas" [7], ya que el cliente requiere estar cambiando la presentación del sitio, por lo que se debe de prever esta situación.
En cuanto a la programación del sitio web se utilizó PHP y Laravel, luego se implementó la funcionalidad del sitio, en la cual se programaron varios puntos entre ellos: se hicieron los links de los botones, las finanzas, la creación de nuevos eventos, y el llenado de varios formularios, así como también el ingreso por medio de usuarios administradores y usuarios normales. En la figura 1 se muestra la página principal de la Asociación Ribs.

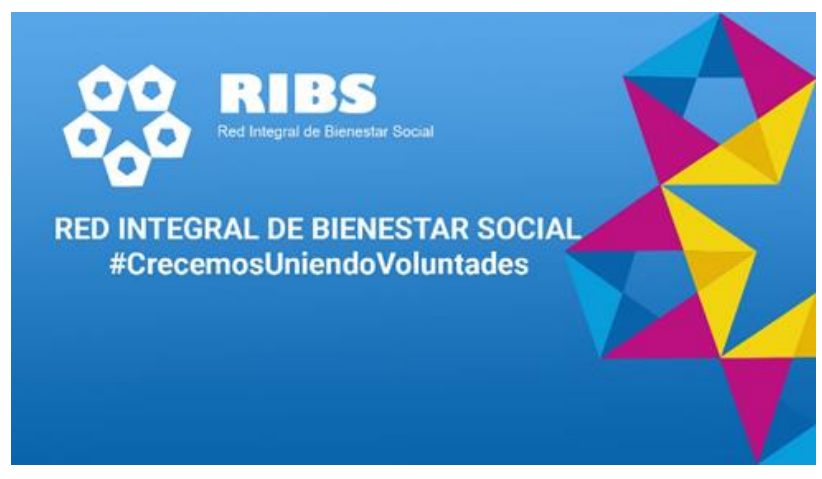

Figura 1 Página Principal

Fuente: Elaboración Propia

\section{Evolución y entrega}

Una vez finalizado un sprint se entrega al cliente el resultado del trabajo. Se devuelve al análisis hasta terminar la pila del producto. [8]. Se realizaron algunas demostraciones preliminares, ya que en la metodología Scrum, hay que entregar el incremento de software al cliente de modo que la funcionalidad que se haya implementado pueda demostrarse al cliente $\mathrm{y}$ éste pueda evaluarla, lo cual fue muy satisfactorio. En ese caso se realizaron 3 sprint y posteriormente se mostró el sitio completo a la asociación Ribs.

Existe para cada asociación un usuario y una contraseña para cada representante de las asociaciones para que puedan acceder a cierta información confidencial. Los demás usuarios pueden acceder solo a la página principal, la cual es informativa, y muestra calendarización de eventos, juntas, convocatorias entre otras informaciones públicas. Se entregó a la asociación Ribs, un manual de usuario en digital, para poder hacer un recorrido por el sitio de manera segura y confiable. En la figura 2 se muestra la pantalla donde se pide el usuario y la contraseña y en base a sus privilegios se mostrará la información asignada según su nivel de usuario. 


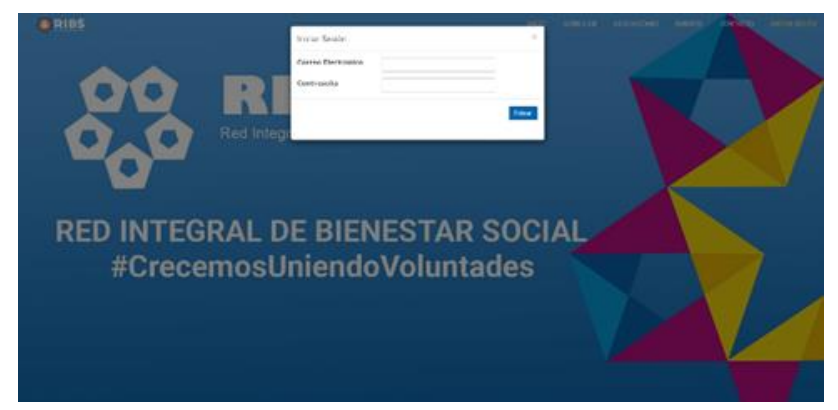

Figura 2 Página de login

Fuente: Elaboración Propia

Al entrar a la página se mostrarán todas las asociaciones con las que se trabaja actualmente como se muestra en la figura 3 .

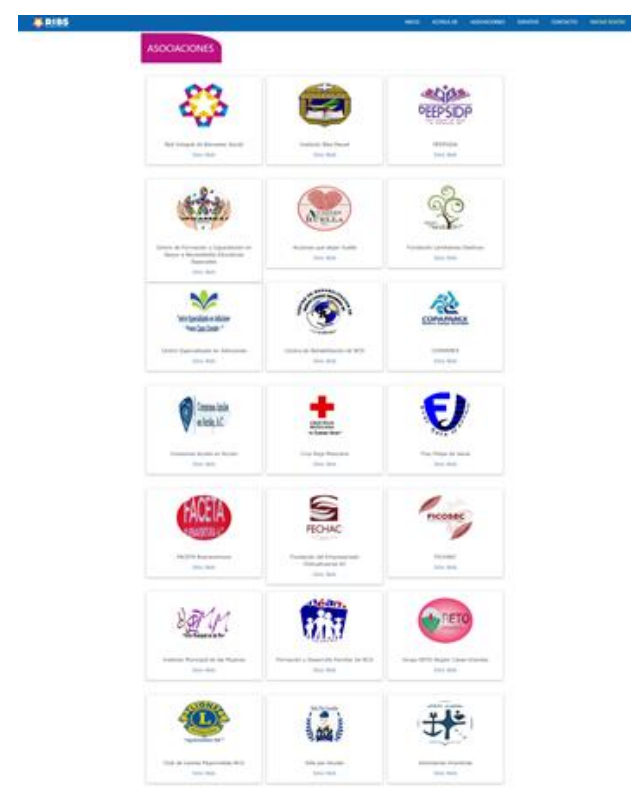

Figura 3 Página de las Asociaciones Fuente: Elaboración Propia

\section{Resultados}

Se analizaron los resultados de las encuestas iniciales de la recolección de la información y se volvieron a retomar las preguntas para volver a realizar los cuestionarios a las mismas asociaciones, pero ahora después de utilizar el sitio web. En la figura 4, se muestra la pregunta sobre ¿el tiempo que se tardan las asociaciones en acceder a la información de entrada y salida de dinero es menor a 48 horas? y las respuestas se muestran en la gráfica, y en la figura 5 se vuelve a mostrar la misma pregunta, pero ahora después de utilizar el sitio web y esto fueron los resultados.

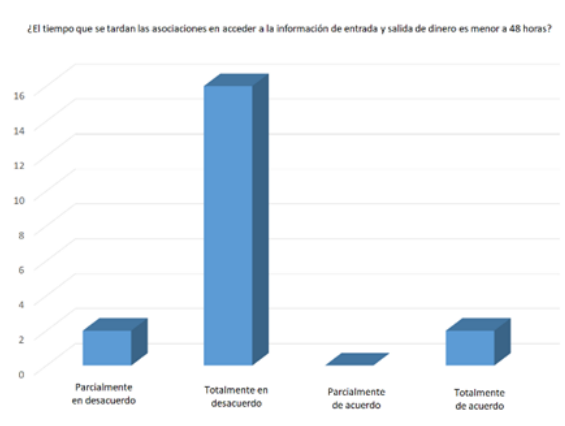

Figura 4 Pregunta antes de la implementación del sitio web

Fuente: Elaboración Propia

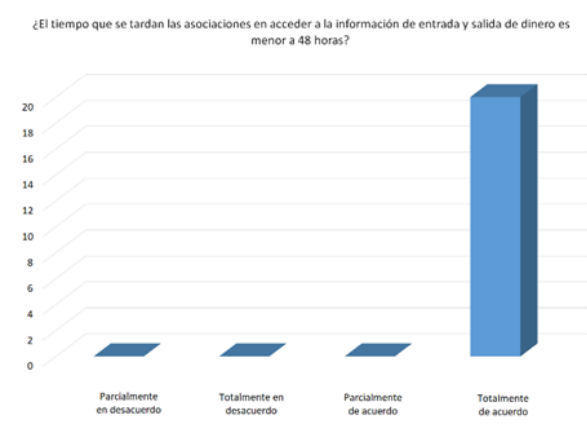

Figura 5 Pregunta después de la implementación del sitio web

Fuente: Elaboración Propia

Como podemos observar, ahora todas las asociaciones están totalmente de acuerdo en que el tiempo que se tardan las asociaciones en acceder a información de entrada y salida de dinero es menor a 48 horas. En la figura 6 , se muestra otra pregunta que se respondió inicialmente en la recolección de la información la cual es: ¿Se puede acceder a la información de transparencia de manera inmediata? y en la figura 7 , la misma pregunta, pero ahora después de utilizar el sitio web.

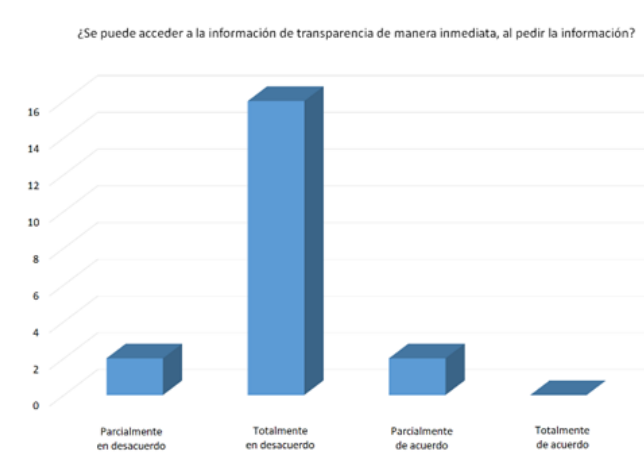

Figura 6 Pregunta antes de la implementación del sitio web

Fuente: Elaboración Propia 


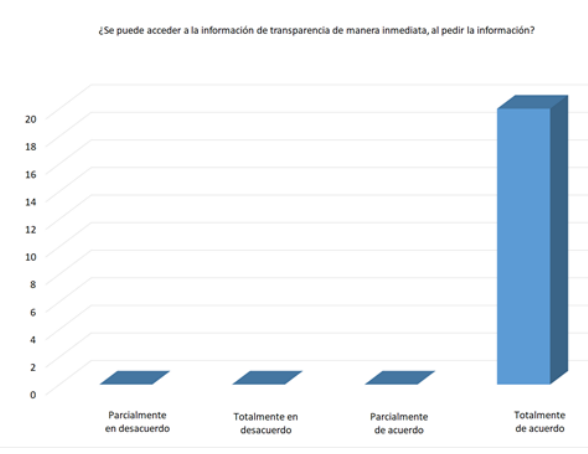

Figura 7 Pregunta después de la implementación del sitio web

Fuente: Elaboración Propia

Como podemos ver los resultados son óptimos, ya que podemos observar que todos están totalmente de acuerdo. El sitio web, ayudo a administrar mejor la información que se maneja en la asociación y a fortalecer los medios electrónicos en la asociación de RIBS, ya que facilitó y agilizó el acceso a la información referente a convocatorias, eventos, juntas y finanzas por lo que ahora se cuenta con un mejor servicio en la difusión y control de la información.

Además de que los alumnos participantes en este proyecto adquirieron experiencia al trabajar con una empresa externa y elaboraron un sitio formal, amigable y seguro para a la asociación, lo cual va a impactar en su desempeño y curriculum personal. A continuación, mostraremos algunas pantallas importantes del sitio web.

En la figura 8, podemos observar la sección donde se publica información para las asociaciones. Del lado izquierdo podemos ver que aparece un menú, el cual contiene cada una de las opciones principales del sitio web.

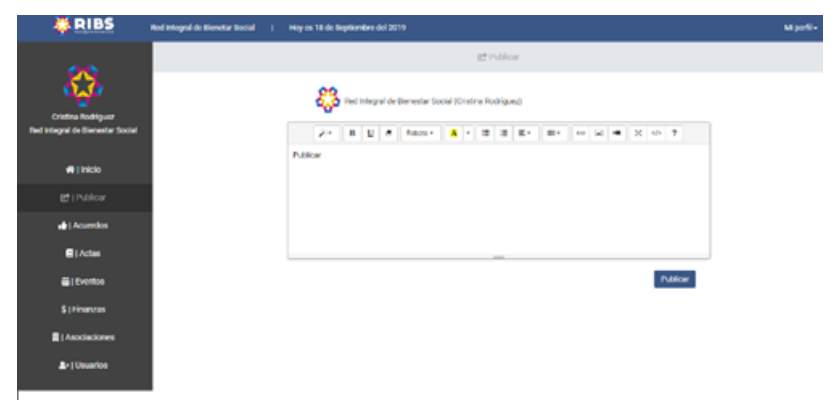

Figura 8 Publicaciones

Fuente: Elaboración Propia

En la figura 9, se muestra la sección donde se almacenan las finanzas de las asociaciones y se publican solamente a las personas autorizadas para verlas y/o imprimirlas.

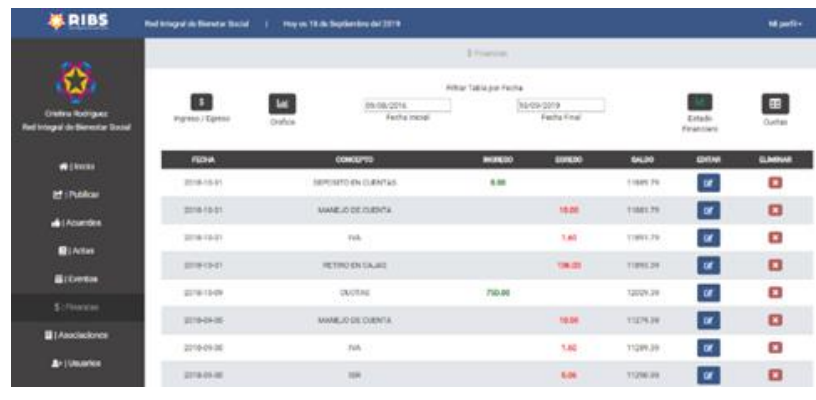

Figura 9 Finanzas

Fuente: Elaboración Propia

\section{Conclusiones}

La información que generan las empresas es muy importante $\mathrm{y}$ tiene un valor muy significativo, ya que forma parte de su crecimiento y evolución, ya que esta información se puede tomar como base para tomar decisiones que afectan directamente a la empresa y a sus clientes, por esta razón es fundamental administrar adecuadamente la información y para esto podemos hacer uso de diversas herramientas tecnológicas que nos pueden ayudar a tener una mejor administración en la información. Por lo que podemos concluir que la implementación del sitio web en la asociación Ribs, es sin duda una gran alternativa para lograr este objetivo ya que este recurso puede ser de gran apoyo ante las grandes demandas que se generan hoy en día por parte de este mundo globalizado.

La asociación Ribs mejoró de manera significativa la realización de las convocatorias, el llenado de actas y el control de las finanzas, ya que si la administradora del sitio publica una nueva convocatoria ya no lo hace por medio de correo y solo lo publica en el sitio web, y al momento que un usuario registrado ingresa a la plataforma le aparecerá una notificación que le dirá sobre que trata la notificación, también fue una parte muy importante el llenado de actas, ya que los datos se almacenan en tablas y al momento de la generación del reporte solo selecciona la información de la base de datos y lo muestra en PDF. Una ventaja de este sitio es que los administradores de las diferentes asociaciones pueden ver el acta de cada reunión, así como descargarla en formato PDF.

\section{Referencias}

[1] Applegate, L., Austin, R. \& Soule, D. (2009). Corporate Information Strategy and Management Text and Cases, 8va. Edición, Singapur: Mc Graw Hill Interamericana. 
[2] Gates, B. (2000). Los Negocios en la Era Digital. 1a. Edición, España: Plaza y Janés Editores.

[3] NewWeb. Motivos para tener mi propio sitio web. Consultado el 3 de septiembre de 2018. Página:

https://www.newwweb.com.mx/index.php/7motivos-para-tener-mi-propio-sitio-web

[4] Tafur, P., Izaguirre, S. (2016). Cómo hacer un proyecto de investigación. Uso de diagramas, matrices y mapas conceptuales. 2da. Edición. Editorial Alfa Omega, p. 189. ISBN: 978-958778-006-2

[5] Martel, A. (2016). Gestión práctica de proyectos con scrum. 3ra. Edición. Editorial: Antonio Martel. ISBN: 978-1517-192-365

[6] Pantaleo, G. (2016). Calidad en el desarrollo de software. 2da. Edición. Editorial Alfa Omega, p. 132. ISBN: $978-987-3832-05-5$

[7]Espinosa M. (2018). Informática Industrial. Primera Edición. Editorial Alfaomega, Marcombo, p. 142. ISBN: 978-607-538-052-0

[8] Escribano, D. (2009). Scrum y los clientes. Obtenido de la página: https://es.slideshare.net/ FlowersInSpace/introduccion-a-scrum-concaso-prctico-1516220.

[9] Salcedo M., Bermúdez P., Flores A. (2018). La transición de un negocio tradicional hacia la digitalización. Revista de Tecnología e Innovación. Vol. $5 . \quad$ No.16. https://ecorfan.org/bolivia/researchjournals/Tec nologia_e_innovacion/vol5num16/Revista_de_ Tecnologia_e_Innovacion_V5_N16_1.pdf

[10] Hernández Sampieri, R., Fernández Collado, C. y Baptista Lucio, M. P. (2014). Metodología de la investigación. México: Mc Graw-Hill.

[11] Baez, A., Arellanes, N., Sosa, A. (2016). Efectividad de la aplicación de metodologías ágiles para el desarrollo de apps móviles. Un caso de estudio [Versión electrónica]. Revista de Sistemas Computacionales y TIC's. Vol.2 No.6 45-66.

[12] Sommerville, Ian. (2005). Ingeniería de Software. Madrid. 7ma. Edición. España: Pearson. ISBN:84-7829-074-5 